\title{
A property of interpolation spaces
}

\author{
By
}

\author{
LeCh Maligranda
}

Let $A_{0}, A_{1}$ and $A_{2}$ be Banach spaces continuously imbedded in some Hausdorff topological vector space, and let $F$ be an interpolation functor. We consider the question: when is it true that

$$
F\left(\left\{A_{0}, A_{1} \cap A_{2}\right\}\right)=F\left(\left\{A_{0}, A_{1}\right\}\right) \cap F\left(\left\{A_{0}, A_{2}\right\}\right) .
$$

Peetre [4] proved that if $\left\{A_{0}, A_{1}\right\}$ is quasi-linearizable pair, i. e., there exist linear operators $V_{0}(t), V_{1}(t)$ (depending on $t>0$ ) such that: $V_{i}(t): A_{0}+A_{1} \rightarrow A_{i}, i=0,1$, $V_{0}(t) a+V_{1}(t) a=a$ and $\left\|V_{0}(t) a\right\|_{A_{0}}+t\left\|V_{1}(t) a\right\|_{A_{1}} \leqq c K\left(t, a: A_{0}, A_{1}\right)$ for $a \in A_{0}+A_{1}$, and if moreover

$$
\left\|V_{1}(t) a\right\|_{A_{2}} \leqq c_{2}\|a\|_{A_{2}} \text { for } a \in A_{2}
$$

then for $a \in\left(A_{0}+A_{1}\right) \cap\left(A_{0}+A_{2}\right)$, we have

$$
K\left(t, a: A_{0}, A_{1} \cap A_{2}\right) \leqq c_{3}\left(K\left(t, a ; A_{0}, A_{1}\right)+K\left(t, a ; A_{0}, A_{2}\right)\right)
$$

From (2) it follows that (1) is true for any $K$-interpolation functor $F=K_{\Phi}$ (in particular, for $F=K_{\theta q}$ ). The couples $\left\{C, C^{1}\right\},\left\{L_{p}, W_{p}^{k}\right\}$ are quasi-linearizable and the couple $\left\{L_{p_{0}}, L_{p_{1}}\right\}, p_{0} \neq p_{1}$ is not quasi-linearizable (cf. [3]). Triebel [5] has given an example of Banach spaces for which equality in (1) does not hold when $F=K_{\theta q}$ or $F=C_{[\theta]}$.

In this note we prove affirmative results in the case of Banach lattices on $(\Omega, \mu)$.

Let $L^{0}(\Omega, \mu)$ be the topological vector space of all measurable functions on a measure space $\Omega$ with a $\sigma$-finite measure $\mu$ (the topology is the one which induces convergence in measure).

A Banach space $X \subset L^{0}(\Omega, \mu)$ is called a Banach lattice on $(\Omega, \mu)$ if $|x| \leqq|y| \quad \mu-$ a.e. on $\Omega, x \in L^{0}(\Omega, \mu)$ and $y \in X$ imply that $x \in X$ and $\|x\|_{X} \leqq\|y\|_{X}$.

Theorem 1. Let $X_{0}, X_{1}$ and $X_{2}$ be Banach lattices on $(\Omega, \mu)$. Then, for $x \in\left(X_{0}+X_{1}\right) \cap\left(X_{0}+X_{2}\right)$, we have

$$
K\left(t, x ; X_{0}, X_{1} \cap X_{2}\right) \leqq 2\left(K\left(t, x ; X_{0}, X_{1}\right)+K\left(t, x ; X_{0}, X_{2}\right)\right) .
$$


Proof. Let $x \in\left(X_{0}+X_{1}\right) \cap\left(X_{0}+X_{2}\right)$. For each $\varepsilon>0$ there exist decompositions $x=x_{0 t}+x_{1 t}=x_{0 t}^{\prime}+x_{2 t}$ with $x_{0 t}, x_{0 t}^{\prime} \in X_{0}$ and $x_{1 t} \in X_{1}, x_{2 t} \in X_{2}$ such that

$$
\begin{aligned}
& \left\|x_{0 t}\right\|_{X_{0}}+t\left\|x_{1 t}\right\|_{X_{1}} \leqq(1+\varepsilon) K\left(t, x ; X_{0}, X_{1}\right) \\
& \left\|x_{0 t}^{\prime}\right\|_{X_{0}}+t\left\|x_{2 t}\right\|_{X_{2}} \leqq(1+\varepsilon) K\left(t, x ; X_{0}, X_{2}\right) .
\end{aligned}
$$

Put $U_{t}=\left\{s \in \Omega:\left|x_{1 t}(s)\right| \leqq\left|x_{2 t}(s)\right| \mu-\right.$ a.e. $\}$ and define $y_{i t}$ by

$$
y_{0 t}(s)=\left\{\begin{array}{l}
x_{0 t}(s), s \in U_{t} \\
x_{0 t}^{\prime}(s), s \in \Omega \backslash U_{t}
\end{array}, \quad y_{1 t}(s)=\left\{\begin{array}{l}
x_{1 t}(s), s \in U_{t} \\
x_{2 t}(s), s \in \Omega \backslash U_{t}
\end{array} .\right.\right.
$$

Then $y_{0 t}+y_{1 t}=x$ and $\left|y_{0 t}\right| \leqq\left|x_{0 t}\right|+\left|x_{0 t}^{\prime}\right|,\left|y_{1 t}\right| \leqq \min \left(\left|x_{1 t}\right|,\left|x_{2 t}\right|\right)$. Furthermore,

and

$$
\begin{aligned}
\left\|y_{0 t}\right\|_{X_{0}} & \leqq\left\|x_{0 t}\right\|_{X_{0}}+\left\|x_{0 t}^{\prime}\right\|_{X_{0}} \leqq(1+\varepsilon) K\left(t, x ; X_{0}, X_{1}\right) \\
& +(1+\varepsilon) K\left(t, x ; X_{0}, X_{2}\right)
\end{aligned}
$$

$$
\begin{aligned}
t\left\|y_{1 t}\right\|_{X_{1} \cap X_{2}} \leqq t \max \left(\left\|x_{1 t}\right\|_{X_{1}},\left\|x_{2 t}\right\|_{X_{2}}\right) \leqq(1+\varepsilon) K\left(t, x ; X_{0}, X_{1}\right) \\
+(1+\varepsilon) K\left(t, x ; X_{0}, X_{2}\right) .
\end{aligned}
$$

Consequently, we obtain

$$
\begin{aligned}
K\left(t, x ; X_{0}, X_{1} \cap X_{2}\right) & \leqq\left\|y_{0 t}\right\|_{X_{0}}+t\left\|y_{1 t}\right\|_{X_{1} \cap X_{2}} \\
& \leqq 2(1+\varepsilon) K\left(t, x ; X_{0}, X_{1}\right)+2(1+\varepsilon) K\left(t, x ; X_{0}, X_{2}\right)
\end{aligned}
$$

and the proof is finished.

Now we will investigate equality (1) for the complex interpolation functors and Banach lattices on $(\Omega, \mu)$.

Let $X_{0}$ and $X_{1}$ be two Banach lattices on $(\Omega, \mu)$ and let $0 \leqq \theta \leqq 1$. We denote by $X_{0}^{1-\theta} X_{1}^{\theta}$ the Calderón space of all $x \in L^{0}(\Omega, \mu)$ such that $|x| \leqq \lambda\left|x_{0}\right|^{1-\theta}\left|x_{1}\right|^{\theta} \mu-$ a.e. on $\Omega$ for some constant $\lambda>0$ and some $x_{i} \in X_{i}$ with $\left\|x_{i}\right\|_{X_{1}} \leqq 1, i=0,1$. We put

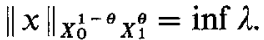

We note that $X_{0}^{1-\theta} X_{1}^{\theta}$ is a Banach lattice on $(\Omega, \mu)$.

Theorem 2. Let $X_{0}, X_{1}$ and $X_{2}$ be Banach lattices on $(\Omega, \mu)$. Then

$$
X_{0}^{1-\theta}\left(X_{1} \cap X_{2}\right)^{\theta}=X_{0}^{1-\theta} X_{1}^{\theta} \cap X_{0}^{1-\theta} X_{2}^{\theta} .
$$

Proof. It is sufficient to prove that

$$
X_{0}^{1-\theta} X_{1}^{\theta} \cap X_{0}^{1-\theta} X_{2}^{\theta} \subset X_{0}^{1-\theta}\left(X_{1} \cap X_{2}\right)^{\theta} .
$$

First we note that if $x_{i} \in X_{i}, i=1,2$, then $\min \left(\left|x_{1}\right|,\left|x_{2}\right|\right) \in X_{1} \cap X_{2}$ and

$$
\left\|\min \left(\left|x_{1}\right|,\left|x_{2}\right|\right)\right\|_{X_{1} \cap X_{2}} \leqq \max \left(\left\|x_{1}\right\|_{X_{1}},\left\|x_{2}\right\|_{X_{2}}\right) \text {. }
$$

Let $x \in X_{0}^{1-\theta} X_{1}^{\theta} \cap X_{0}^{1-\theta} X_{2}^{\theta}$ with the norm $\leqq 1$ and let $\varepsilon>0$. Then there exist $x_{0}, x_{0}^{\prime} \in X_{0}$, $x_{i} \in X_{i}, i=1,2$ with norms $\leqq 1$ and

$$
|x| \leqq(1+\varepsilon)\left|x_{0}\right|^{1-\theta}\left|x_{1}\right|^{\theta}, \quad|x| \leqq(1+\varepsilon)\left|x_{0}^{\prime}\right|^{1-\theta}\left|x_{2}\right|^{\theta} \quad \mu-\text { a.e. }
$$


We have

$$
\begin{aligned}
|x| & \leqq(1+\varepsilon) \min \left(\left|x_{0}\right|^{1-\theta}\left|x_{1}\right|^{\theta},\left|x_{0}^{\prime}\right|^{1-\theta}\left|x_{2}\right|^{\theta}\right) \\
& \leqq(1+\varepsilon) \min \left[\max \left(\left|x_{0}\right|,\left|x_{0}^{\prime}\right|\right)^{1-\theta}\left|x_{1}\right|^{\theta}, \max \left(\left|x_{0}\right|,\left|x_{0}^{\prime}\right|\right)^{1-\theta}\left|x_{2}\right|^{\theta}\right] \\
& =(1+\varepsilon) \max \left(\left|x_{0}\right|,\left|x_{0}^{\prime}\right|\right)^{1-\theta} \min \left(\left|x_{1}\right|,\left|x_{2}\right|\right)^{\theta} .
\end{aligned}
$$

Hence $x \in X_{0}^{1-\theta}\left(X_{1} \cap X_{2}\right)^{\theta}$ and the norm is $\leqq 2(1+\varepsilon)$.

From Theorem 2 and Calderón's results (see [2], p. 125) it follows that if Banach lattices $X_{0}, X_{1}$ and $X_{2}$ on $(\Omega, \mu)$ have absolutely continuous [Fatou] norms then (1) is true for the lower [upper] complex interpolation functor $F=C_{[\theta]}\left[=C^{[\theta]}\right]$.

\section{References}

[1] J. BERGH and J. LÖFSTRÖM, Interpolation Spaces. Berlin-Heidelberg-New York 1976.

[2] A. P. Calderón, Intermediate spaces and interpolation, the complex method. Studia Math. 24, $113-190$ (1964).

[3] J. PeETre, Zur Interpolation von Operatorenräumen. Arch. Math. 21, $601-608$ (1970).

[4] J. PEeTRE, Über den Durchschnitt von Interpolationsräumen. Arch. Math. 25, 511-513 (1974).

[5] H. Triebel, Eine Bemerkung zur nicht-kommutativen Interpolation. Math. Nachr. 69, 57-60 (1975).

[6] H. Triebel, Interpolation Theory. Function Spaces. Differential Operators. Berlin 1978.

Eingegangen am 21. 8. 1984

Anschrift des Autors:

Lech Maligranda

Institute of Mathematics

Polish Academy of Sciences

Mielżyńskiego 27/29

61-725 Poznań, Poland 